RESEARCH ARTICLE

\title{
High Intracranial Pressure and Retinitis Pigmentosa
}

\author{
Habib Dezhagah, $M D^{1,2,3^{*}}$, Hamid Sajjadi, MD ${ }^{4,5,6}$ and Mohammad-Ali Abtahi, MD \\ ${ }^{1}$ Director, Private Eye Clinic, Apadana Building, Kianpars, Ahvaz, Iran \\ ${ }^{2}$ Department of Ophthalmology, Apadana Hospital, Ahvaz, Iran \\ ${ }^{3}$ Razi Eye Center, Palizvani Street, Gandi, Tehran, Iran \\ ${ }^{4}$ Director, San Jose Eye and Laser Medical Center, San Jose, California, USA \\ ${ }^{5}$ Director, Department of Ophthalmology, Acacia Medical Center, Dubai, UAE \\ ${ }^{6}$ Director, Department of Ophthalmology, Iranian Red Crescent Hospital, Dubai, UAE \\ ${ }^{7}$ Sadra Eye Clinic, Isfahan, Iran
}

*Corresponding author: Habib Dezhagah, MD, Director, Private Eye Clinic, Apadana Building, Kianpars, Ahvaz, Iran; Department of Ophthalmology, Apadana Hospital, Ahvaz, Iran; Razi Eye Center, Palizvani Street, Gandi, Tehran, Iran, Tel: $+989161132607$

\begin{abstract}
Objective: We evaluated our RP patients for signs and symptoms of increased intracranial pressure (ICP).

Methods: After history taking and complete eye examination, optical coherence tomography (OCT) of the optic nerve and retina, and then, laboratory tests and magnetic resonance imaging (MRI) of the brain and orbit were done. Those whose findings were in favor of increased ICP were sent to a neurologist for a lumbar puncture (LP). Forty- four patients were eligible to enter the study, but only 23 patients (10 males) completed the work-up and underwent LP.

Results: The mean age was 41 years. Only three patients $(13 \%)$ were obese and $65 \%$ had a headache. All patients $(100 \%)$ showed two or more signs of increased ICP in MRI. Three patients $(13 \%)$ had an elevated level of vitamin A, but $56.5 \%$ and $48 \%$ of patients were deficient in vitamins $D$ and B12, respectively. The checked mean ICP was $250 \mathrm{mmH}_{2} \mathrm{O}$ in decubitus position.

Conclusions: In conclusion, we suggest that RP patients should have a worked-up for high ICP, and serum levels of vitamins $D$ and B12, which deficiency of each of them may lead to secondary optic nerve damage.
\end{abstract}

\section{Keywords}

Retinitis pigmentosa, Intracranial pressure, Pseudotumor cerebri

\begin{abstract}
Abbreviation
OCT: Optical Coherence Tomography; MRI: Magnetic Resonance Imaging; LP: Lumbar Puncture; SD: Standard Deviation; BMI: Body Mass Index; ICP: Intracranial Pressure; ng/ml: Nanogram/milliliter; pg/ml: Picogram/milliliter; RP: Retinitis Pigmentosa; NFL: Nerve Fiber Layer
\end{abstract}

\section{Introduction}

Retinitis pigmentosa is the name commonly given to a group of disorders characterized over many years by progressive loss of visual field, night blindness, and degeneration of the retina [1]. It is estimated that it affects about 1.6 million people worldwide [2]. The first manifestation of the disease, typically arising during early adolescence, is night blindness (nyctalopia) due to the impaired rod photoreceptors, followed by progressive death of these cells. Subsequently, patients experience a restriction of the visual field (tunnel vision), because of further loss of rods in the peripheral retina, where these cells predominate. Later, patients undergo a progressive decline of visual acuity in the central field, and impaired chromatic discrimination, due to the gradual demise of cones [1].

Alternatively, visual acuity can remain normal, even in individuals with advanced retinitis pigmentosa with a small island of the remaining central visual field, or it

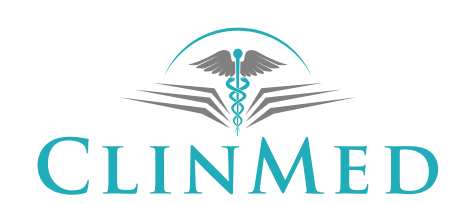

INTERNATIONAL LIBRARY
Citation: Dezhagah H, Sajjadi H, Mohammad-Ali A (2020) High Intracranial Pressure and Retinitis Pigmentosa. Int J Ophthalmol Clin Res 7:123. doi.org/10.23937/2378-346X/1410123

Accepted: December 29, 2020: Published: December 31, 2020

Copyright: (C) 2020 Dezhagah $\mathrm{H}$, et al. This is an open-access article distributed under the terms of the Creative Commons Attribution License, which permits unrestricted use, distribution, and reproduction in any medium, provided the original author and source are credited. 
can be lost early during the disorder. Acute vision loss was also reported in retinitis pigmentosa patients [3]. Other possible symptoms in retinitis pigmentosa patients include chronic light flashes, headaches, paresthesia, and vision, varying from day to day and under different situations [4].

These symptoms were also reported in idiopathic intracranial hypertension [5]. Actually, the similarities in the symptoms between retinitis pigmentosa and idiopathic intracranial hypertension are very striking. Idiopathic intracranial hypertension, or pseudo tumor cerebri, is a challenging condition with raised intracranial pressure in the absence of an identifiable cause [6]. The most common symptom at the presentation in idiopathic intracranial hypertension patients is a headache [7]. It usually presents in around $93 \%$ of patients at the time of diagnosis, usually being constant or occurring daily or nearly daily [8]. Monocular or binocular transient visual obscurations varying from slight blurring to total loss of light perception are seen in up to $72 \%$ of patients with idiopathic intracranial hypertension [9]. Photopsia and continuous blurred vision with normal visual acuity are other frequent visual complaints [10]. Even acute visual loss has been reported in increased intracranial pressure [11].

Pulse-synchronous tinnitus is another commonly reported symptom of idiopathic intracranial hypertension ( $58 \%$ of patients) and is often described as a unilateral "whooshing" sound by patients and may be exacerbated by positional changes and relieved by jugular compression [12]. Tinnitus is reported in $31.5 \%$ of retinitis pigmentosa patients [13].

Visual loss, an only serious complication of idiopathic intracranial hypertension, usually has a good prognosis, but in untreated patients can lead to permanent visual loss [14].

The optic disc elevation in retinitis pigmentosa has been reported before. David, et al. [15] reported a 19-year-old man, diagnosed with pseudo tumor cerebri due to the visual field defect, optic disc elevation, and intracranial pressure of $350 \mathrm{mmH}_{2} \mathrm{O}$; later, a diagnosis of retinitis pigmentosa was made according to the fundus examination and electroretinography finding. Although the authors claimed that the disc drusen was mistaken for papilledema, and the visual field defect was due to retinal degeneration, instead of high intracranial pressure, which was a spurious measurement. Another report of a 44-year-old man, a known case of retinitis pigmentosa, who had bilateral papilledema and his intracranial pressure, was $195 \mathrm{~mm} \mathrm{H}_{2} \mathrm{O}$ [16].

Previously, Sajjadi, et al. described specific optical coherence tomography patterns that were highly suggestive of increased intracranial pressure [17]. As they claimed, these patterns are useful in screening those patients who are suspected to have idiopathic intracranial hypertension. Because of similarities of signs and symptoms of retinitis pigmentosa and idiopathic intracranial hypertension, and importance of early detection of idiopathic intracranial hypertension to prevent irreversible visual loss, especially in retinitis pigmentosa patients, whose visions already are impaired, we conducted this study on patients with the previous diagnosis of retinitis pigmentosa to check if they are associated with idiopathic intracranial hypertension.

\section{Materials and Methods}

\section{Study design and population}

This prospective study was conducted on consecutive patients with the previous diagnosis of retinitis pigmentosa, referred to the Ophthalmology clinic between 2018 and 2019. The study was approved by the local Ethical Committee and all participants signed an informed consent before enrollment.

\section{Inclusion criteria}

All patients with a retinitis pigmentosa diagnosis, with informed consent and optical coherence tomography in favor of raised intracranial pressure, were included in the study.

\section{Exclusion criteria}

A history of previous ocular trauma, ocular surgery (except for cataract surgery) or ocular co-morbidity except for retinitis pigmentosa, and presence of intracranial tumor or a history of an intracranial surgery.

\section{Methods}

After history taking, all patients received complete ophthalmologic examination, including visual acuity testing, slit-lamp, and fundus examination, color fundus photography, and optical coherence tomography imaging (Figure 1). Because all cases had optical coherence tomography in favor of raised intracranial pressure, after discussing the probability of pseudo tumor cerebri with them, they were asked to do a laboratory blood test (including vitamins A, D, and B12) and magnetic resonance imaging of the brain and orbit (without contrast). The compact disc of magnetic resonance imaging was reviewed by a neuro-ophthalmologist (Sajjadi, H.), and after calculating the signs of the raised intracranial pressure in magnetic resonance imaging, patients were referred to the neurologist for a lumbar puncture and a check of intracranial pressure. The intracranial pressure was checked in a lateral decubitus position. In those patients who had high intracranial pressure, the medication was started. The medications we prescribed included acetazolamide, topiramate and/or spironolactone. The patients were asked to come in the first follow-up after one month. During a follow-up visit, a complete eye exam was done and the patients were asked if medication they use has any side effects. Then, the patients asked to come for further follow-up every three months. 

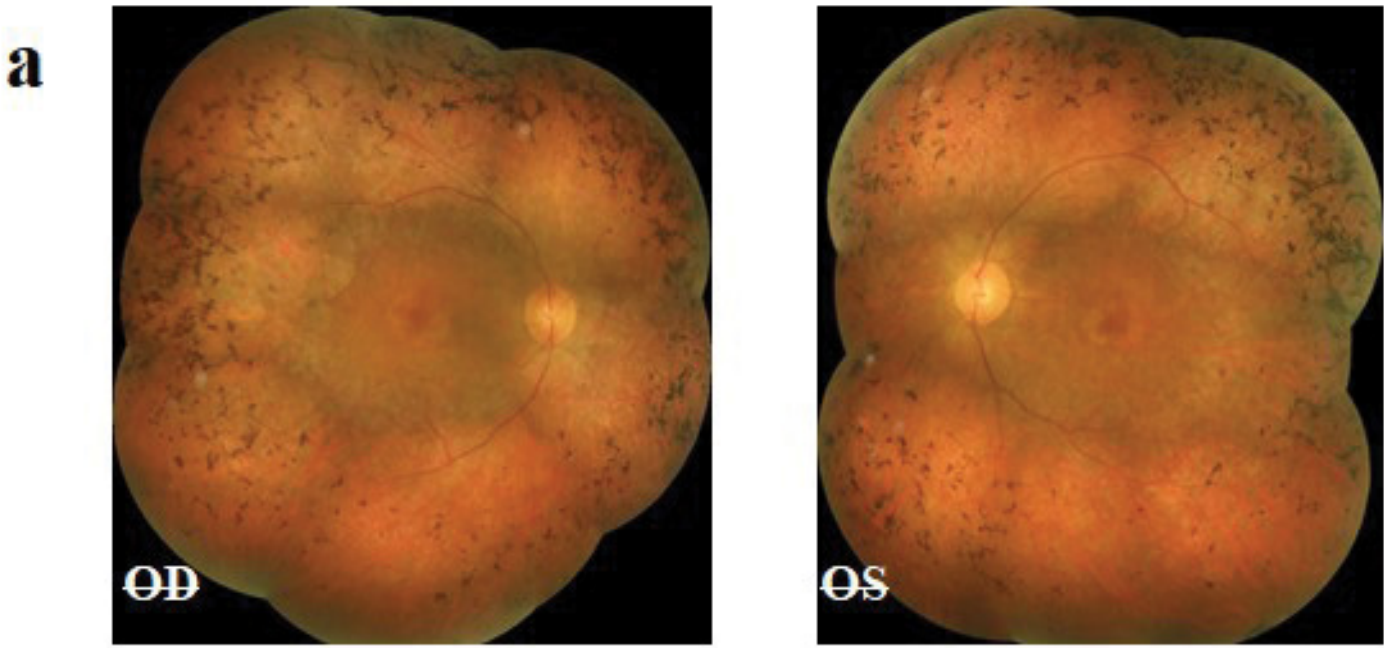

b

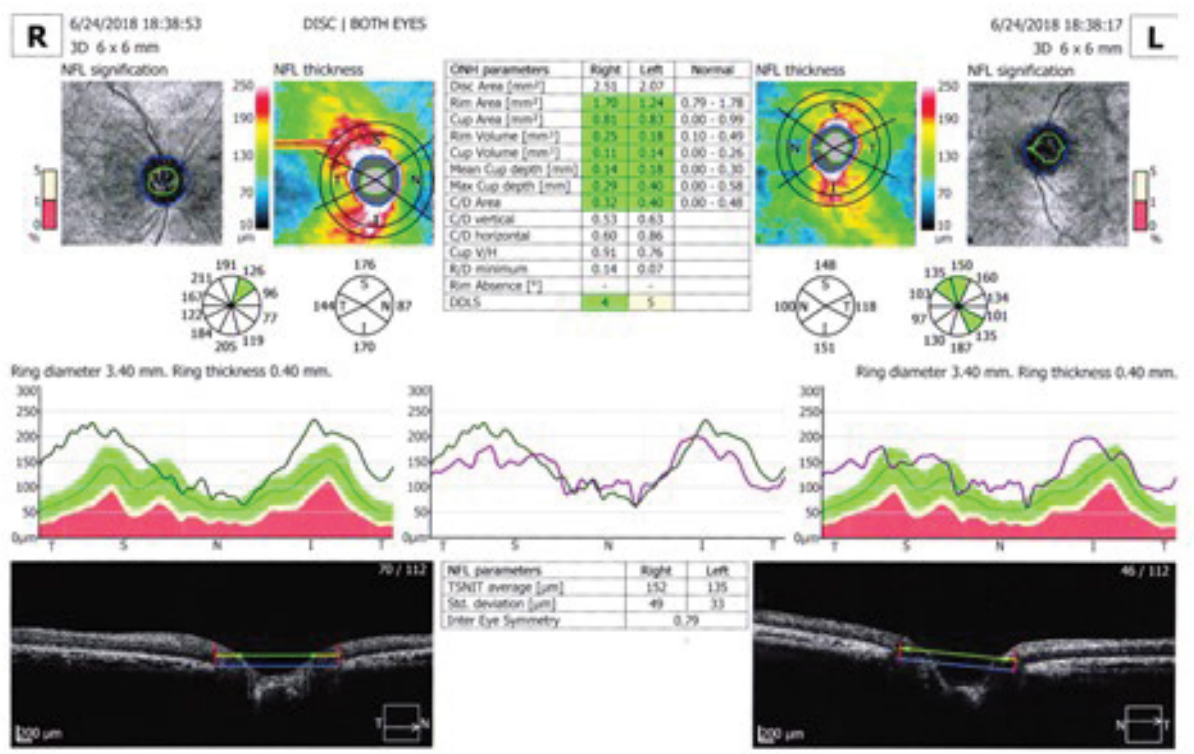

$\mathbf{c}$
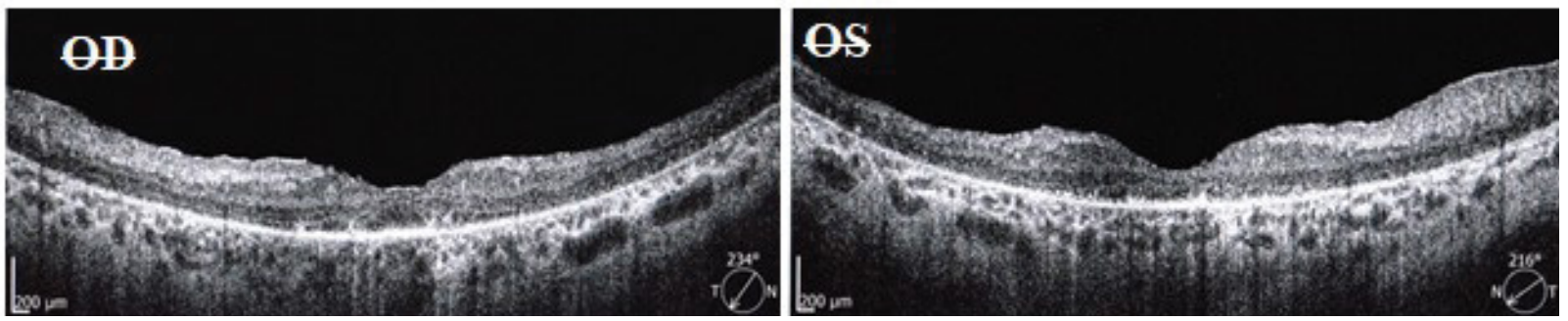

Figure 1: a) Fundus photos of a patient with retinitis pigmentosa; b) Optical coherence tomography (OCT) of the optic nerve heads of the same patient, showing significant thickening of the nerve fiber layer; c) OCT of the macula of the same patient.

\section{Statistical analysis}

Data were presented using descriptive statistics such as mean, standard deviation, frequency, and percentage. Then, $t$-test and chi-square tests were used to estimate the population parameters. A $P$-value of $<0.05$ was considered statistically significant. Data were analyzed using SPSS version 20.

\section{Results}

Forty-four retinitis pigmentosa patients were visited and completed the initial eye examination and optical coherence tomography imaging during an 18-month pe- riod. Despite a complete explanation of the probability of high intracranial pressure, according to the optical coherence tomography finding, 20 patients $(45.5 \%)$ neither refer to the neurologist nor undergo lumbar puncture. In one patient, the lumbar puncture was unsuccessful. Finally, the study was completed with 23 cases $(52 \%)$ and 21 cases (48\%) were excluded.

Ten patients (43\%) were males. Mean age and mean body mass index of the patients were $41 \pm 12.7$ years (range, 26-67 year) and $27 \pm 4.5$ (range, 21.3-40.2), respectively. Only three patients (13\%) were obese (body mass index more than 30$)$, and nine (39\%) had a body 
Table 1: Demographics of Patients with Retinitis Pigmentosa.

Variables

Sex, number (\%)

History of Headache, Number (\%)

Age (y), Mean $\pm S^{*}$ (Range)

$\mathrm{BMI}^{\dagger}\left(\mathrm{Kg} / \mathrm{m}^{2}\right)$, Mean \pm SD (Range)

MRI ${ }^{\ddagger}$ findings: Minor signs of increased ICP§

Vitamin A Serum level, number (\%)

Vitamin D, serum level, Mean \pm SD (Range), $\mathrm{ng} / \mathrm{m}^{\prime \prime} \mid$

Vitamin $B_{12}$, serum level, Mean \pm SD (Range), pg/m \#

ICP, Mean \pm SD (Range), $\mathrm{mmH}_{2} \mathrm{O}^{* *}$

\section{Values}

$10(43 \%)$

\begin{tabular}{|l|l|}
\hline Male & $10(43 \%)$ \\
Female & $13(57 \%)$ \\
\hline Yes & $15(65 \%)$ \\
No & $8(35 \%)$ \\
& $41 \pm 12.7(25-67)$ \\
\hline & $27 \pm 4.5(21.3-40.2)$ \\
\hline Normal & $100 \%$ of patients showed 2 or more signs \\
\hline Abnormal & $20(85 \%)$ \\
& $3(15 \%)$ \\
& $21.4 \pm 12.6(5.8-39)$ \\
\hline & $271 \pm 150(45-573)$ \\
\hline & $250 \pm 81(170-490)$ \\
\hline
\end{tabular}

SD*: Standard Deviation; BMI+: Body Mass Index; MRI: Magnetic Resonance Imaging; ICP\$: Intracranial Pressure; ng/mIll: Nanogram/milliliter; $\mathrm{pg} / \mathrm{ml}^{\#}$ : Picogram/milliliter; $\mathrm{mmH}_{2} \mathrm{O}^{* *}$ : Millimeter of Water.

a
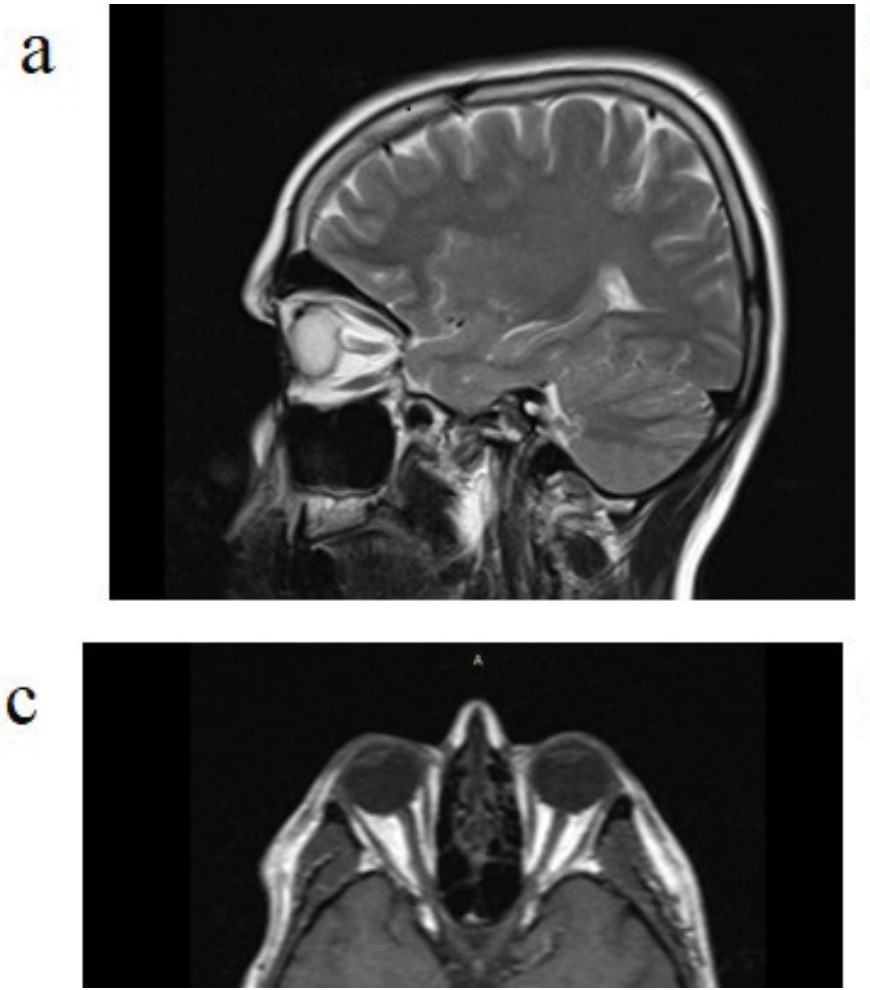

$\mathrm{b}$

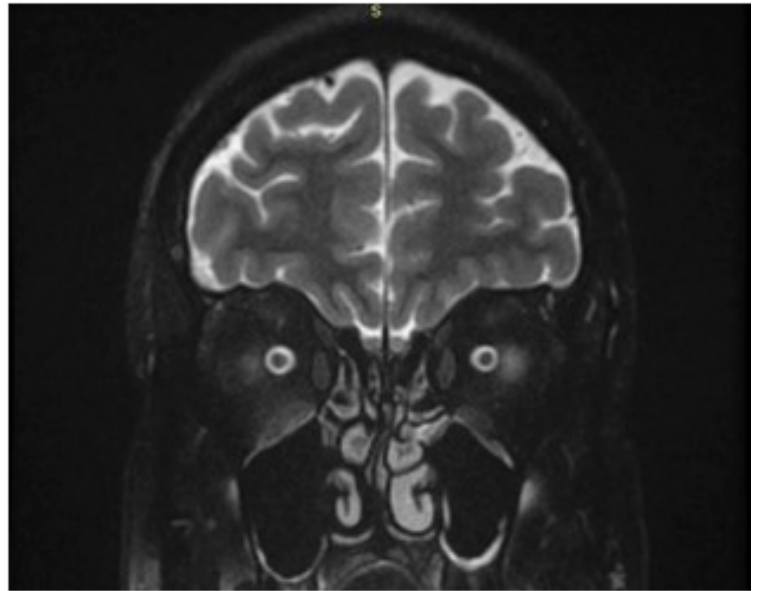

d

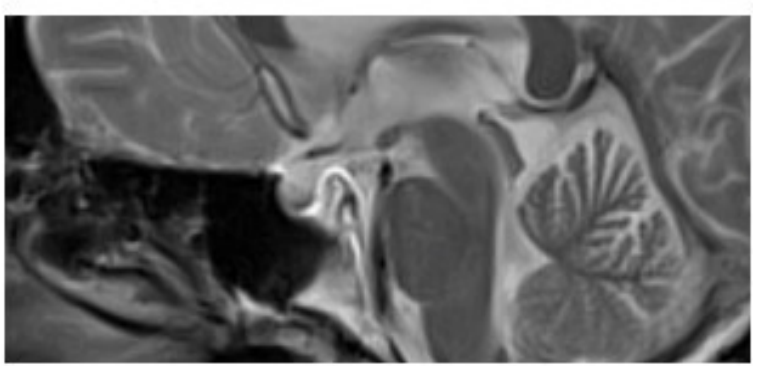

Figure 2: Some signs of increased intracranial pressure in MRI of study patients; a) Optic nerve head protrusion; b) Optic nerve sheath diameter increase; c) Posterior scleral flattening; d) Partial empty sella.

mass index in the range of 21-25. The rest of the body mass indexes were $25-30$. Only $65 \%$ of the patients had a headache, which was relieved after starting the treatment (Table 1).

In optical coherence tomography imaging, all patients showed the peripapillary nerve fiber layer thickening (Figure 1).

According to Sajjadi, et al., about $75 \%$ of the patients had patterns 1 or 2 . Another optical coherence tomography finding was a ganglion cell layer loss, which was present in all patients. Also, two patients (8\%) had cystoid macular edema in both eyes, which finally, when the patients were seen in follow-up visits, and after starting acetazolamide, cystoid macular edema was resorbed completely.

Regarding the signs of increased intracranial pressure in magnetic resonance imaging, $100 \%$ of patients showed two or more of these signs, including posterior scleral flattening, empty or partially empty sella, optic nerve tortuosity, optic nerve head protrusion, increased optic nerve sheath diameter and descended cerebellar tonsil (Figure 2). 
Only three patients (13\%) had elevated serum levels of vitamin $A$, and the others had normal levels. Alternatively, most of our patients had less than normal serum levels of vitamins D3 and B12. Mean levels of vitamins $\mathrm{D} 3$ and $\mathrm{B} 12$ were $21.4 \pm 12.6 \mathrm{ng} / \mathrm{ml}$ (range 5.8-39) and $271 \pm 150 \mathrm{pg} / \mathrm{ml}$ (range 45-573), respectively. About $56.5 \%$ of patients had a deficiency of vitamin D3 (serum level less than $20 \mathrm{ng} / \mathrm{ml}$ ), 8.6\% had insufficient vitamin D3 (serum level between $21-30 \mathrm{ng} / \mathrm{ml}$ ), and $48 \%$ were deficient in vitamin B12 (serum level less than $180 \mathrm{pg} /$ $\mathrm{ml}$ ). Also, $30 \%$ of patients had a deficiency of both vitamins D3 and B12.

The intracranial pressure was checked by lumbar puncture in lateral decubitus position by a neurologist. Mean intracranial pressure was $250 \pm 81 \mathrm{mmH}_{2} \mathrm{O}$ (range, $170-490)$. Nine patients (39\%) had intracranial pressure in the range of $17-20 \mathrm{mmH}_{2} \mathrm{O}$. Also, the cerebrospinal fluid composition in all patients was normal.

\section{Discussion}

To our knowledge, this is the first objective study investigating raised intracranial pressure in retinitis pigmentosa patients.

Almost $45.5 \%$ of our patients did not complete the work-up. Despite a complete explanation of the possibility of the raised intracranial pressure, and the need to do requested work-up, many of our patients referred to another physician (ophthalmologist and/or neurologist) to get a second opinion. Also, increased intracranial pressure in retinitis pigmentosa patients is not a wellknown entity to our colleagues. During this process, as patients said, the possibility of the raised intracranial pressure is denied by physicians and some of them were dissuaded from doing work-up of the raised intracranial pressure, especially lumbar puncture. Perhaps, the main reason is that our patients lost to follow-up.

Some authors use the mean age of diagnosis of retinitis pigmentosa, and some others use the patients' mean age of the time of the study. Because determining the exact age of onset is often difficult, [18] and our patients could not give a clear history of the disease onset; we used their age at the first exam and calculate the mean age. The patients mean age was higher than the patients mean age in the similar studies, [19] because we used age at the examination time, and our patients were known cases of retinitis pigmentosa, meaning the diagnosis was made years before, so it is acceptable that the mean age of the exam is higher than the mean age of diagnosis of the disease. Also, our patients' access to eye care centers may be affected by lower socioeconomic class and cultural status of patients. So, we believe that our patients referred to us with delay.

To our knowledge, no study has checked body mass index in retinitis pigmentosa patients. In a study conducted on 33 Indian patients with a diagnosis of pseudo tumor cerebri, most of the patients were of ideal weight
[20]. Sixty-five percent of our patients had a headache. In one study, the most common symptom of 500 retinitis pigmentosa patients was a headache, which was present in $53.3 \%$ of patients [4].

All patients showed the peripapillary nerve fiber layer thickening in optical coherence tomography. Previous reports also confirmed the peripapillary nerve fiber layer thickening in papilledema or optic nerve head elevation [21]. Papilledema in idiopathic intracranial hypertension is a consequence of the increased perineural pressure with subsequent axonal optic nerve head swelling. Increased peripapillary nerve fiber layer thickness and total retinal thickness in optical coherence tomography are proposed as useful markers for quantifying papilledema severity [22]. Eren $\mathrm{Y}$, et al. believed that optical coherence tomography can contribute to the diagnosis of idiopathic intracranial hypertension, by providing reliable data on optical cup volume, optical disc and rim area, and greater retinal nerve fiber layer thickness [23]. All patients had ganglion cell layer thinning. This finding in optical coherence tomography of retinitis pigmentosa patients has been reported in previous studies [24]. Also, in another report, the average ganglion cell-inner plexiform layer thickness in retinitis pigmentosa patients was less than normal controls [25].

The cystoid macular edema frequency in retinitis pigmentosa has been reported as $10-50 \%$ [26]. In the present study, the cystoid macular edema frequency was only $8 \%$, relatively low and closed to the lowest reported values compared to the previous reports. The reason for the cystoid macular edema low frequency in the present study may be due to differences in the study protocols and methodology compared with the previous studies. Besides, our patients were relatively older and less complicated compared to previous studies.

All patients in their magnetic resonance imaging showed two or more signs of increased intracranial pressure. The findings included posterior scleral flattening, optic nerve tortuosity, empty sella, partially empty sella, the increased optic nerve sheath diameter, cerebellar tonsil descending and optic nerve head protrusion. There are many neuroimaging studies in retinitis pigmentosa patients, but, to our knowledge, no study investigated the increased intracranial pressure signs in the retinitis pigmentosa patients yet. In a study, [27] specific visual cortical gray matter loss in retinitis pigmentosa patients associated with their visual function profile was found. Kitajima, et al. found that the calcarine fissure increased in width in retinitis pigmentosa patients, notably within the anterior and middle regions that normally represent peripheral vision [28].

Only $13 \%$ of patients had elevated vitamin A serum levels, but none of them had used a regular amount of supplementary vitamin A before. We checked vitamin A serum levels in our patients looking for any elevation of it because some authors found a relationship between 
high intracranial pressure and elevated serum level of vitamin A [29]. So, in our retinitis pigmentosa patients who had elevated intracranial pressure, vitamin A elevated serum levels are less likely to be the cause.

A relatively high fraction of our patients $(65 \%)$ had vitamin D3 deficiency or insufficiency. It is widely known that vitamin $D$ receptors have been found in neurons and glial cells, and their highest expression is in the hippocampus, hypothalamus, thalamus and subcortical gray matter nuclei, and substantia nigra [30]. Sajjadi, et al. found a relationship between low vitamin $D$ and high intracranial pressure. Zaki, et al. reported a case of a five-month-old female infant with vitamin $D$ deficiency rickets, who presented with pseudo tumor cerebri [31]. Our finding regarding low vitamin D3 level and high intracranial pressure is consistent with above-mentioned studies.

Vitamin D deficiency in older patients is associated with reduced mean ganglion cell complex thickness, indicating an early stage of optic nerve damage, before retinal nerve fiber layer loss [30]. Particularly, Annweiler, et al., have reported that vitamin D deficiency is associated with smaller volumes of optic chiasm in older adults [32]. The reduced mean of ganglion cell complex thickness and small optic chiasma can result in optic atrophy. Our patients were not checked for the optic chiasm size, but all of them had a ganglion cell loss, explaining why they had some degree of optic atrophy. Forty-eight percent of our patients had vitamin B12 deficiencies. Vitamin B12 deficiency is an important cause of optic neuropathy, [33] and some reports showed a vitamin B12 deficiency can present with optic disc edema and/or optic atrophy [34]. None of our patients had visible optic disc edema. About $30 \%$ had both vitamin D3 and B12 deficiency. Overall, a higher percentage of vitamin $\mathrm{D}$ and/or vitamin B12 deficiency in our patients could be a reason for the optic atrophy.

We considered the upper normal limit of intracranial pressure as $200 \mathrm{mmH}_{2} \mathrm{O}$ [35]. Thirty-nine percent of our patients had intracranial pressure in the range of 170 $200 \mathrm{mmH}_{2} \mathrm{O}$. We believe that even these intracranial pressures are in the range of upper normal limit, but it may be considered too high in patients with already damaged optic nerve due to the damage of photoreceptors and/or optic atrophy secondary to vitamins D or B12 deficiency.

In conclusion, most retinitis pigmentosa patients have high intracranial pressure, and vitamins $D$ and B12 deficiency, affecting the function of the already damaged optic nerve in retinitis pigmentosa. So, every retinitis pigmentosa patient should have a work-up for the elevated intracranial pressure and serum levels of vitamins D and B12.

\section{Acknowledgments}

The authors gratefully acknowledge all those who helped us in this project.

\section{Sources of Funding}

No funds were used to perform the study and the authors funded the budget of the project.

\section{Disclosures of Conflict of Interest}

None.

\section{Authors Declarations}

The study was conducted according to the Declaration of Helsinki.

\section{References}

1. Guadagni V, Novelli E, Piano I, Gargini C, Strettoi E (2015) Pharmacological approaches to retinitis pigmentosa: A laboratory perspective. Prog Retin Eye Res 48: 62-81.

2. Ontario HQ (2016) Retinal prosthesis system for advanced retinitis pigmentosa: A health technology assessment update. Ont Health Technol Assess Ser 17: 1-62.

3. Patil-Chhablani $P$, Tyagi $M$, Kekunnaya $R$, Narayanan $R$ (2015) Acute unilateral vision loss with optic disc oedema in retinitis pigmentosa. BMJ Case Rep 2015: bcr2015210869.

4. Heckenlively JR, Yoser SL, Friedman LH, Oversier JJ (1988) Clinical findings and common symptoms in retinitis pigmentosa. Am J Ophthalmol 105: 504-511.

5. Round R, Keane JR (1988) The minor symptoms of increased intracranial pressure: 101 patients with benign intracranial hypertension. Neurology 38: 1461-1464.

6. Friedman DI (2014) The pseudotumor cerebri syndrome. Neurol Clin 32: 363-396.

7. Bruce BB, Biousse V, Newman NJ (2011) Update on idiopathic intracranial hypertension. Am J Ophthalmol 152: 163-169.

8. Craig J, Mulholland D, Gibson J (2001) Idiopathic intracranial hypertension: Incidence, presenting features and outcome in Northern Ireland (1991-1995). Ulster Med J 70: 31-35.

9. Skau M, Brennum J, Gjerris F, Jensen R (2006) What is new about idiopathic intracranial hypertension? An updated review of mechanism and treatment. Cephalalgia 26: 384-399.

10. Wall M, George D (1991) Idiopathic intracranial hypertension: A prospective study of 50 patients. Brain 114: 155180.

11. Thambisetty M, Lavin PJ, Newman NJ, Biousse V (2007) Fulminant idiopathic intracranial hypertension. Neurology 68: 229-232.

12. Degnan A, Levy L (2011) Pseudotumor cerebri: Brief review of clinical syndrome and imaging findings. AJNR Am J Neuroradiol 32: 1986-1993.

13. Iwasaki S, Maruyama Y, Hotta Y, Hashimoto Y, Nagura M (2004) Survey in to the prevalence of hearing loss in patients diagnosed with retinitis pigmentosa. Int Ophthalmol 25: 277-282.

14. Corbett JJ, Savino PJ, Thompson HS, Kansu T, Schatz NJ, et al. (1982) Visual loss in pseudotumor cerebri: follow-up of 57 patients from five to 41 years and a profile of 14 patients with permanent severe visual loss. Arch Neurol 39: 461-474.

15. Heidemann DG, Beck RW (1987) Retinitis pigmentosa. A mimic of neurologic disease. Surv Ophthalmol 32: 45-51. 
16. Villa AM, Anderson SF, Abundo RE (1997) Bilateral disc edema in retinitis pigmentosa. Optom Vis Sci 74: 132-137.

17. Sajjadi F, Khoshnevisan M, Doane J, Sajjadi H (2017) New predictive value of optical coherence tomography analysis in the diagnosis of idiopathic intracranial hypertension. J Contemp Med Sci 3: 197-207.

18. Verbakel SK, van Huet RA, Boon CJ, den Hollander Al, Collin RW, et al. (2018) Non-syndromic retinitis pigmentosa. Prog Retin Eye Res 66: 157-186.

19. Tsujikawa M, Wada $Y$, Sukegawa $M$, Sawa $M$, Gomi F, et al. (2008) Age at onset curves of retinitis pigmentosa. Arch Ophthalmol 126: 337-340.

20. Pal A, Sengupta P, Biswas D, Sen C, Mukherjee A, et al (2019) Pattern of idiopathic intracranial hypertension in Indian population. Ann Indian Acad Neurol 22: 47-51.

21. Rebolleda G, Munoz-Negrete FJ (2009) Follow-up of mild papilledema in idiopathic intracranial hypertension with optical coherence tomography. Invest Ophthalmol Vis Sci 50: 5197-5200.

22. Huang-Link Y-M, Al-Hawasi A, Oberwahrenbrock T, Jin Y-P (2015) OCT measurements of optic nerve head changes in idiopathic intracranial hypertension. Clin Neurol Neurosurg 130: 122-127.

23. Eren Y, Kabatas N, Guven H, Comoglu S, Gurdal C (2019) Evaluation of optic nerve head changes with optic coherence tomography in patients with idiopathic intracranial hypertension. Acta Neurol Belg 119: 351-357.

24. Liu G, Li H, Liu X, Xu D, Wang F (2016) Structural analysis of retinal photoreceptor ellipsoid zone and postreceptor retinal layer associated with visual acuity in patients with retinitis pigmentosa by ganglion cell analysis combined with OCT imaging. Medicine 95: e5785.

25. Humayun MS, Prince M, de Juan E, Barron Y, Moskowitz $M$, et al. (1999) Morphometric analysis of the extramacular retina from postmortem eyes with retinitis pigmentosa. Invest Ophthalmol Vis Sci 40: 143-148.
26. Bakthavatchalam M, Lai FH, Rong SS, $\mathrm{Ng}$ DS, Brelen ME (2018) Treatment of cystoid macular edema secondary to retinitis pigmentosa: a systematic review. Surv Ophthalmol 63: 329-339.

27. Machado AR, Pereira AC, Ferreira F, Ferreira S, Quendera B, et al. (2017) Structure-function correlations in retinitis pigmentosa patients with partially preserved vision: A voxel-based morphometry study. Sci Rep 7: 1-10.

28. Kitajima M, Korogi Y, Hirai T, Hamatake S, Ikushima I, et al. (1997) MR changes in the calcarine area resulting from retinal degeneration. AJNR Am J Neuroradiol 18: 1291-1295.

29. Chen J, Wall M (2014) Epidemiology and risk factors for idiopathic intracranial hypertension. Int Ophthalmol Clin 54: $1-11$.

30. Moretti R, Morelli ME, Caruso P (2018) Vitamin D in neurological diseases: A rationale for a pathogenic impact. Int $J$ Mol Sci 19: 2245.

31. Zaki SA, Lad V, Abdagire N (2013) Vitamin D deficiency rickets presenting as pseudotumor cerebri. J Neurosci Rural Pract 2013, 4: 464-466.

32. Annweiler C, Beauchet O, Bartha R, Graffe A, Milea D, et al. (2013) Association Between Serum 25-Hydroxyvitamin D Concentration and Optic Chiasm Volume. J Am Geriatr Soc 61: 1026-1028.

33. Chu C, Scanlon P (2011) Vitamin B12 deficiency optic neuropathy detected by asymptomatic screening. BMJ Case Rep.

34. Petramfar P, Hosseinzadeh F, Mohammadi SS (2016) Pseudo-Foster Kennedy Syndrome as a Rare Presentation of Vitamin B12 Deficiency. Iran Red Crescent Med J 18: e24610.

35. Sugerman HJ, Felton III WL, Sismanis A, Kellum JM, DeMaria EJ, et al. (1999) Gastric surgery for pseudotumor cerebri associated with severe obesity. Ann Surg 229: 634. 